\title{
Environmental exposures as a risk factor for fibrolamellar carcinoma
}

\author{
Rondell P Graham ${ }^{1}$, John R Craig ${ }^{2}$, Long Jin ${ }^{1}$, Andre M Oliveira ${ }^{1}$, John R Bergquist ${ }^{3}$, \\ Mark J Truty ${ }^{3}$, Taofic Mounajjed ${ }^{1}$, Patricia T Greipp ${ }^{1}$ and Michael S Torbenson ${ }^{1}$ \\ ${ }^{1}$ Department of Laboratory Medicine and Pathology, Mayo Clinic, Rochester, MN, USA; ${ }^{2}$ Laennec Liver \\ Pathology Society and ${ }^{3}$ Department of Surgery, Mayo Clinic, Rochester, MN, USA
}

\begin{abstract}
Fibrolamellar carcinoma was first described in 1956. Subsequent large studies failed to identify cases before 1939 (the start of the World War II). This finding, combined with the presence of aryl hydrocarbon receptors on the tumor cells, have suggested that fibrolamellar carcinomas may be caused by environmental exposures that are new since World War II. To investigate this possibility, the surgical pathology files before 1939 were reviewed for hepatocellular carcinomas resected in young individuals. Two cases of fibrolamellar carcinoma were identified, from 1915 to 1924. The diagnosis of fibrolamellar carcinoma was confirmed at the histologic, ultrastructural and proteomic levels. These two fibrolamellar carcinoma cases clarify a key aspect of fibrolamellar carcinoma biology, reducing the likelihood that these tumors result exclusively from post World War II environmental exposures.

Modern Pathology (2017) 30, 892-896; doi:10.1038/modpathol.2017.7; published online 3 March 2017
\end{abstract}

Fibrolamellar carcinoma is a unique primary hepatic malignancy characterized by a predilection for young individuals without underlying liver disease. ${ }^{1-3}$ The distinct histologic findings include large eosinophilic cells, which frequently have pale bodies and hyaline bodies. The tumor also characteristically shows abundant intratumoral fibrosis. The distinctive nature of fibrolamellar carcinoma extends to the protein level. Fibrolamellar carcinomas are routinely positive for CK7 (ref. 4) and their increased lysosomal content leads to positive CD68 immunostaining. ${ }^{5}$ Fibrolamellar carcinomas also overexpress anterior gradient-2 (ref. 6) and ornithine aminotransferase, ${ }^{7}$ while showing reduced or absent LFABP staining. ${ }^{8}$ At the molecular level, fibrolamellar carcinomas show a specific DNAJB1-PRKACA fusion transcript in almost all bona fide cases. ${ }^{7,9}$

The etiological risk factors for fibrolamellar carcinomas are unknown, as they arise in livers without underlying liver disease and without fibrosis. However, as one possible clue, Craig and Edmonson noted that no cases of fibrolamellar carcinoma were identified in large autopsy studies ${ }^{10,11}$ or in the consultation practice of Dr Edmonson before World

Correspondence: Professor MS Torbenson, MD, Department of Laboratory Medicine and Pathology, Mayo Clinic, Rochester, MN 55905, USA.

E-mail: Torbenson.michael@mayo.edu

Received 18 October 2016; revised 23 December 2016; accepted 26

December 2016; published online 3 March 2017
War II (1939-1945). Furthermore, Oikawa et al ${ }^{12}$ suggested that the incidence of fibrolamellar carcinoma is increasing, perhaps related to the development of the plastics industry during and after World War II.

The goal of this study is to further investigate the presence/absence of fibrolamellar carcinomas before 1939, using morphological findings and additional testing to confirm cases.

\section{Materials and methods}

With IRB approval, the pathology archives at Mayo Clinic from 1905 to 1939 were reviewed for surgical resections of hepatocellular carcinoma in individuals $<50$ years of age. Original slides were examined (RPG and MST) and cases that were likely fibrolamellar carcinoma based on the original slides were selected for further study. The clinical charts were reviewed. Immunohistochemistry for HepPar1 (clone OCH1E5; predilute; Ventana, Tucson, AZ, USA), CK7 (clone OV-TL 12/30; 1:100; Dako, Santa Clara, CA, USA) and CD68 (clone KP1; 1:100; Dako) was performed using heat-induced epitope retrieval and standard laboratory methods.

Ultrastructural examination was performed on both cases using formalin-fixed paraffin-embedded tissues. The sections were reviewed on a FEI Tecnai 12 model electron microscope. Fluorescent in situ hybridization for PRKACA rearrangement and 


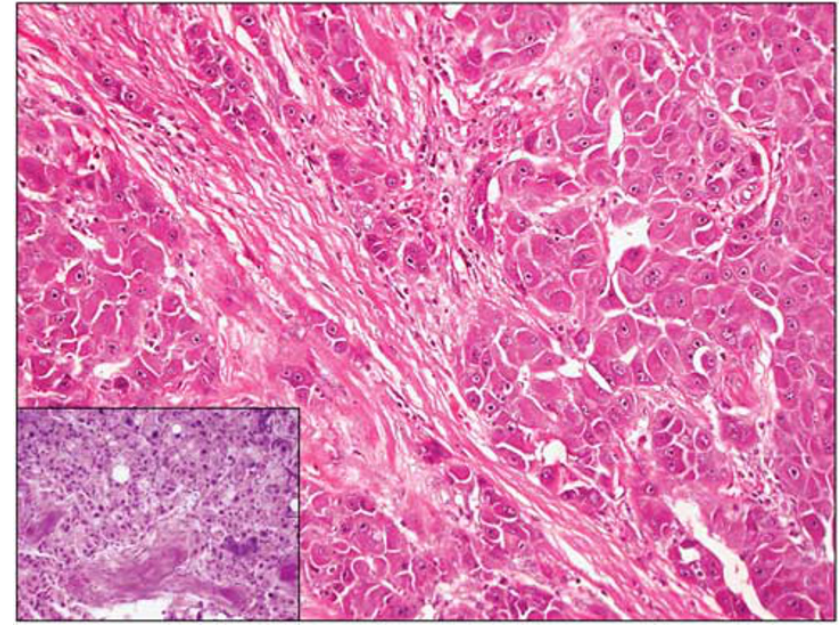

Figure 1 The photomicrograph shows a typical fibrolamellar carcinoma composed of oncocytic neoplastic cells with prominent macronucleoli. The neoplastic cells form trabecula, which are separated by bands of fibrosis (original magnification $\times 200$ ). Inset: the original toluidine stained slide from 1915 revealed the same histologic features and focal intratumoral steatosis.

RT-PCR for DNAJB1-PRKACA were performed using formalin-fixed, paraffin-embedded tissues, as previously described. ${ }^{9}$ Finally, laser capture microdissection and mass spectrometry-based proteomic analysis were performed on formalin-fixed, paraffin-embedded tissue sections as previously described. $^{8}$

\section{Results}

Case 1 A 39-year-old man who worked as a fence builder presented with a history of abdominal pain in 1915. Clinical examination revealed only right upper quadrant tenderness. Gynecomastia was not present. The persistence of the pain led to surgical intervention. From the operative notes, a hen eggsized mass was found in the right lobe of the liver, close to the fundus of the gallbladder. The mass was excised and sent for pathological examination. The patient was followed for 9 years after surgery, during which no recurrence or metastatic disease was documented. He was lost to follow-up thereafter.

On review of the original slides, the tumor was composed of large eosinophilic cells with macronucleoli, separated by parallel arrays of lamellar fibrosis. Focally, the neoplastic cells showed steatosis (inset of Figure 1). A recut H\&E confirmed the classic histological findings of fibrolamellar carcinoma (Figure 1). Immunostains were focally positive for HepPar1 and CK7 but the CD68 immunostain failed, as evidenced by no staining of Kupffer cells. FISH and RT-PCR were unsuccessful because of failure of hybridization and poor quality RNA, respectively. However, mass spectrometry-based proteomics was successful and confirmed that the tumor was hepatocellular, with detection of

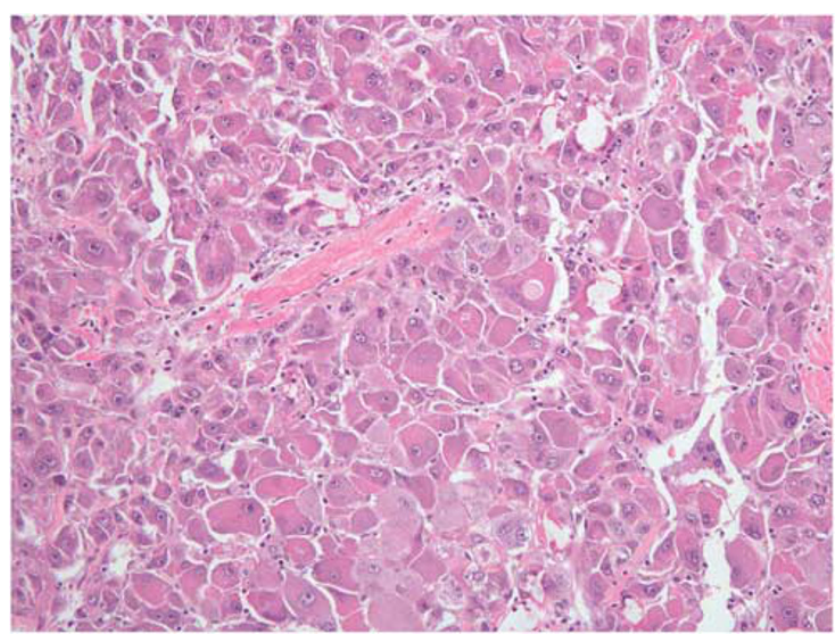

Figure 2 This case from 1924 showed the same histologic features and is characteristic of fibrolamellar carcinoma. A pale body is shown in the center of the image. Pale bodies are seen in almost half of fibrolamellar carcinomas.

Arginase. Importantly, multiple classic markers of fibrolamellar carcinoma were also detected: CK7, anterior gradient-2 and ornithine aminotransferase, confirming the diagnosis of fibrolamellar carcinoma.

Case 2 A 68-year-old man who worked as a banker presented in 1924 with a clinical history of abdominal pain and a liver mass excision, 29 years prior, which was not available for review. At laparotomy, an advanced liver mass was identified, with extension to the lesser curvature of the stomach, adenopathy and ascites. An incisional biopsy was performed. On clinical follow-up, the patient died of disease $<11$ months after presentation.

Histologic sections showed the typical elements of fibrolamellar carcinoma: monotonous large neoplastic cells with granular eosinophilic cytoplasm, prominent macronucleoli and bands of intratumoral fibrosis (Figure 2). Occasional pale bodies and pink bodies were identified. CK7 was focally positive. RT-PCR and FISH were unsuccessful because of nucleic acid degradation. However, mass spectrometry-based proteomics was informative, detecting arginase, CK7, anterior gradient-2 and ornithine aminotransferase.

\section{Electron Microscopy Findings}

Ultrastructural analysis of both cases showed similar features. The tumor cells showed abundant cytoplasm rich in mitochondria (Figure 3a). The membranes of the endoplasmic reticulum were abundant and formed concentric whorls (Figure 3b) with geometric/fingerprint-like (Figure 3c) structures. In case 2, internalized canaliculi lined by long microvilli were seen within the cytosol of neoplastic hepatocytes and were surrounded by thick ectoplasm (data not shown). These findings are not entirely specific, but are in line with previous reports 

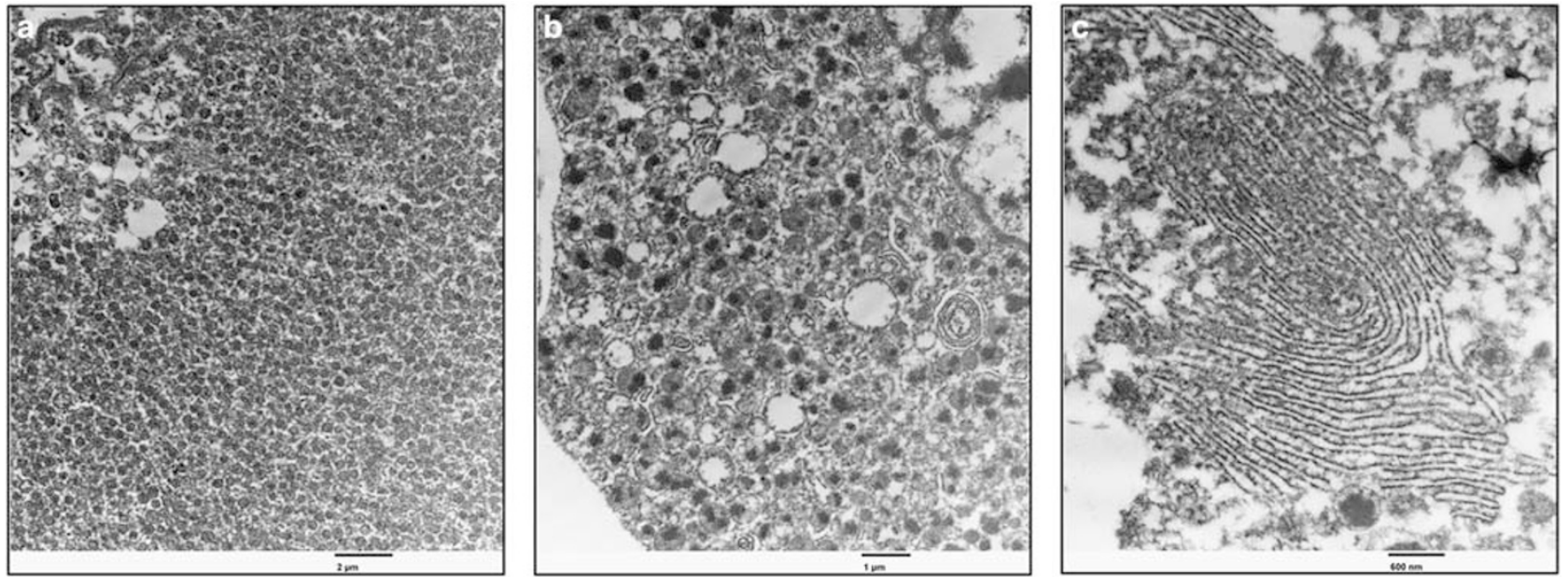

Figure 3 (a) Numerous mitochondria were seen in the cytoplasm of tumor cells from both cases. (b) Concentric whorls of the endoplasmic reticulum are shown in this image. This is a feature reported in fibrolamellar carcinoma. (c) Geometric structures formed by the abundant endoplasmic reticulum are another ultrastructural characteristic of fibrolamellar carcinoma and was seen in these cases.

of ultrastructural findings in fibrolamellar carcinoma from paraffin-embedded tissues., ${ }^{1,2,13-17}$

\section{Discussion}

Fibrolamellar carcinoma was first described by Edmondson ${ }^{18}$ in 1956 as a neoplasm affecting a 14-year-old girl. Craig et $a l^{1}$ refined the unique clinical and histologic characteristics of fibrolamellar carcinoma, including the major histological findings of large polygonal cells with abundant eosinophilic cytoplasm, large nucleoli and prominent intratumoral fibrosis. Craig et $a l^{1}$ also provided the name fibrolamellar carcinoma and described the propensity for fibrolamellar carcinoma to affect individuals between 5 and 35 years of age. The distinctive histologic features of fibrolamellar carcinoma were further emphasized by Berman et $a 1^{19}$ in 1980 and others in review articles since then. ${ }^{20-24}$

The etiology for fibrolamellar carcinoma continues to be a major unresolved question. Early studies of thousands of autopsy and consultation cases found no examples of fibrolamellar carcinoma before World War II. ${ }^{1,10,11}$ This suggested that fibrolamellar carcinoma may be associated with environmental risk factors introduced after the World War II. ${ }^{12}$ This interesting finding was followed by a subsequent observation that tumor cells expressed aryl hydrocarbon receptors, ${ }^{12}$ suggesting a potential role for the plastics industry, which expanded rapidly after 1945. However, the findings in the current study significantly reduce the likelihood that fibrolamellar carcinomas are driven by environmental exposures unique to the post World War II economy, as we describe the first known cases of fibrolamellar carcinoma occurring before World War II, in 1915 and 1924. As a result of the importance of this observation for understanding potential risk factors, the diagnosis was carefully validated by both morphological findings and protein-based studies with supporting ultrastructural examination results. However, these findings do not exclude a role for other potential environmental risk factors. In addition, our results are not able to address the question of whether the frequency of fibrolamellar carcinomas has changed before and after World War II.

The etiology of fibrolamellar carcinomas remains one of the key unsolved questions regarding this tumor. There is a defining molecular feature found in almost all fibrolamellar carcinomas but not in conventional hepatocellular carcinomas, as fibrolamellar carcinomas have deletions on the short arm of chromosome 19 that leads to a DNAJB1-PRKACA fusion gene..$^{7,9,12}$ The etiology for fibrolamellar carcinoma must cause this novel oncogenic genomic event. In general, the known mechanisms for the formation of oncogenic gene fusions include interchromosomal rearrangements, inversions and deletions. ${ }^{25-27}$ The two main prerequisites for formation of a fusion gene by any of these mechanisms are close spatial proximity of the gene partners in the nucleus and the formation of double-stranded DNA breaks. ${ }^{25,26}$ In the case of fibrolamellar carcinoma, the 400-kb separation between the two genes meets the former prerequisites. Double-strand DNA breaks may occur due to homologous recombination errors, the presence of the partner genes at recombination hot spots in the genome, topoisomerase II toxins/ poisons or failure of mechanisms of repair of doublestrand DNA breaks. ${ }^{25,26}$ Patients with inherited syndromes associated with the failure to repair double-stranded DNA breaks (eg, Nijmegen syndrome) have not been reported with fibrolamellar carcinoma. Furthermore, although recipients of topoisomerase inhibitors are at increased risk for translocation-associated leukemias ${ }^{25}$ no fibrolamellar carcinomas have been reported in this clinical 
context. Study of chromosome 19 has revealed no recombination hot spots, making this possible mechanism seem unlikely for fibrolamellar carcinoma. ${ }^{28}$ However, chromosome 19 does show increased levels of homology relative to other chromosomes in the genome, which may make it susceptible to homologous recombination errors. ${ }^{28}$ These errors underlie deletion and duplication syndromes $^{29}$ and would be concordant with the formation of DNAJB1-PRKACA by an interstitial deletion. However, this possibility would need to be tested before making a conclusion concerning the mechanism for double-strand breakage in DNAJB1PRKACA formation. Nevertheless, existing data suggest that recurrent fusion genes are not random events. $^{27}$

The environmental agent(s) responsible for the genomic events giving rise to the formation of DNAJB1-PRKACA remain(s) to be identified but there are some etiologies which have been excluded. Fibrolamellar carcinomas are not associated with chronic viral hepatitis $\mathrm{C}$ or $\mathrm{B}$, alcohol use, iron overload, aflatoxin, or other factors that drive risk in conventional hepatocellular carcinoma. In keeping with this, the background livers are essentially normal at the microscopic level, lacking significant inflammation or fibrosis. ${ }^{30}$ There have been occasional reports documenting the presence of hepatitis $\mathrm{B}$ viral proteins or DNA in fibrolamellar carcinoma, ${ }^{31-33}$ but this finding appears to be one of chance, given the high world-wide prevalence of chronic hepatitis B infection, and overall there are no data to suggest hepatitis B as an etiological agent.

No histological precursor lesions to fibrolamellar carcinoma have been identified and there is no evidence for a 'field effect', as can be seen in conventional hepatocellular carcinoma where the background liver shows chronic disease. Fibrolamellar carcinomas can rarely co-occur with type 1 hepatocellular adenomas (HNF1 $\alpha$-inactivated), but they clearly do not develop out of hepatic adenomas. ${ }^{8}$ Similarly, old notions of possible malignant transformation of focal nodular hyperplasia ${ }^{34}$ have been disproven.

Fibrolamellar carcinomas are well known for their propensity to occur in younger individuals, with $80 \%$ of all cases presenting between ages 10 and $35 .{ }^{21}$ Yet, fibrolamellar carcinomas do not occur in neonates or toddlers, making in utero exposures leading to the tumor less likely.

Animal models provide an informative avenue into our understanding of disease processes and can provide important insights into tumorigenesis. Unfortunately, there are no animal models of fibrolamellar carcinoma. This is in contrast to conventional hepatocellular carcinomas, for which there are dozens of animal models. ${ }^{35-37}$ In these models, exposure to chemicals or chronic viral infection induces development of conventional hepatocellular carcinoma. However, no mutagen exposure has yielded a fibrolamellar carcinoma to date. Nonetheless, rare spontaneous fibrolamellar carcinoma has been reported in other mammals, ${ }^{38}$ suggesting models could yet be developed which may advance our understanding of the etiologic event(s) in fibrolamellar carcinoma.

In conclusion, fibrolamellar carcinoma is a unique translocation-associated hepatocellular carcinoma, which was first recognized in $1956 .{ }^{18}$ Early large studies failed to identify fibrolamellar carcinomas from before World War II. ${ }^{10,11}$ These observations and the finding of aryl hydrocarbon receptors on the tumor cells suggested the possibility that environmental exposures after World War II could be responsible for causing fibrolamellar carcinoma. ${ }^{12}$ The two cases of fibrolamellar carcinoma characterized in this study confirm that the genomic events responsible for formation of the oncogenic DNAJB1PRKACA fusion gene in fibrolamellar carcinoma preceded World War II. Further work is needed to identify the agent(s) responsible for genomic events leading to the formation of the oncogenic DNAJB1PRKACA fusion gene.

\section{Acknowledgments}

We gratefully acknowledge the excellent technical work of the Cytogenetics and Proteomics Core laboratories and the diligent support of research coordinator Janis Donovan in completion of this study. We also thank the Division of Anatomic Pathology for research funding.

\section{Disclosure/conflict of interest}

The authors declare no conflict of interest.

\section{References}

1 Craig JR, Peters RL, Edmondson HA, et al. Fibrolamellar carcinoma of the liver: a tumor of adolescents and young adults with distinctive clinico-pathologic features. Cancer 1980;46:372-379.

2 Torbenson M. Fibrolamellar carcinoma: 2012 update. Scientifica 2012;2012:15.

3 Kakar S, Burgart LJ, Batts KP, et al. Clinicopathologic features and survival in fibrolamellar carcinoma: comparison with conventional hepatocellular carcinoma with and without cirrhosis. Mod Pathol 2005;18: 1417-1423.

4 Ward SC, Huang J, Tickoo SK, et al. Fibrolamellar carcinoma of the liver exhibits immunohistochemical evidence of both hepatocyte and bile duct differentiation. Mod Pathol 2010;23:1180-1190.

5 Ross HM, Daniel HD, Vivekanandan P, et al. Fibrolamellar carcinomas are positive for CD68. Mod Pathol 2011;24:390-395.

6 Vivekanandan P, Micchelli ST, Torbenson M. Anterior gradient-2 is overexpressed by fibrolamellar carcinomas. Hum Pathol 2009;40:293-299.

7 Honeyman JN, Simon EP, Robine N, et al. Detection of a recurrent DNAJB1-PRKACA chimeric transcript in 
fibrolamellar hepatocellular carcinoma. Science 2014; 343:1010-1014.

8 Graham RP, Terracciano LM, Meves A, et al. Hepatic adenomas with synchronous or metachronous fibrolamellar carcinomas: both are characterized by LFABP loss. Mod Pathol 2016;29:607-615.

9 Graham RP, Jin L, Knutson DL, et al. DNAJB1-PRKACA is specific for fibrolamellar carcinoma. Mod Pathol 2015;28:822-829.

10 Edmondson HA, Steiner PE. Primary carcinoma of the liver: a study of 100 cases among 48,900 necropsies. Cancer 1954;7:462-503.

11 Craig JR, Johnson L, Lundberg GD, et al. An autopsy survey of clinical and anatomic diagnoses associated with alcoholism. Arch Pathol Lab Med 1980;104: $452-455$.

12 Oikawa T, Wauthier E, Dinh TA, et al. Model of fibrolamellar hepatocellular carcinomas reveals striking enrichment in cancer stem cells. Nat Commun 2015;6:8070.

13 Kanai T, Takabayashi T, Kawano Y, et al. A case of postoperative recurrence of fibrolamellar hepatocellular carcinoma with increased vitamin B12 binding capacity in a young Japanese female. Jpn J Clin Oncol 2004;34:346-351.

14 Terracciano LM, Tornillo L, Avoledo P, et al. Fibrolamellar hepatocellular carcinoma occurring 5 years after hepatocellular adenoma in a 14-year-old girl: a case report with comparative genomic hybridization analysis. Arch Pathol Lab Med 2004;128:222-226.

15 Payne CM, Nagle RB, Paplanus SH, et al. Fibrolamellar carcinoma of liver: a primary malignant oncocytic carcinoid? Ultrastruct Pathol 1986;10:539-552.

16 Sato S, Masuda T, Oikawa H, et al. Bile canaliculi-like lumina in fibrolamellar carcinoma of the liver: a lightand electron-microscopic study and three-dimensional examination of serial sections. Pathol Int 1997;47: 763-768.

17 Andreola S, Audisio RA, Lombardi L. A light microscopic and ultrastructural study of two cases of fibrolamellar hepatocellular carcinoma. Tumori 1986; 72:609-616.

18 Edmondson HA. Differential diagnosis of tumors and tumor-like lesions of liver in infancy and childhood. AMA J Dis Child 1956;91:168-186.

19 Berman MM, Libbey NP, Foster JH. Hepatocellular carcinoma. Polygonal cell type with fibrous stroma-an atypical variant with a favorable prognosis. Cancer 1980;46:1448-1455.

20 Paradis V. Histopathology of hepatocellular carcinoma. Recent Results Cancer Res 2013;190:21-32.

21 Munshaw S, Hwang HS, Torbenson M, et al. Laser captured hepatocytes show association of butyrylcholinesterase gene loss and fibrosis progression in hepatitis C-infected drug users. Hepatology 2012;56: $544-554$.

22 Torbenson M. Review of the clinicopathologic features of fibrolamellar carcinoma. Adv Anat Pathol 2007;14: 217-223.

23 Kwee HG. Fibrolamellar hepatocellular carcinoma. Am Fam Physician 1989;40:175-177.

24 Vecchio FM. Fibrolamellar carcinoma of the liver: a distinct entity within the hepatocellular tumors. A review. Appl Pathol 1988;6:139-148.

25 Aplan PD. Causes of oncogenic chromosomal translocation. Trends Genet 2006;22:46-55.

26 Zheng J. Oncogenic chromosomal translocations and human cancer (review). Oncol Rep 2013;30:2011-2019.

27 Mitelman F, Johansson B, Mertens F. The impact of translocations and gene fusions on cancer causation. Nat Rev Cancer 2007;7:233-245.

28 Grimwood J, Gordon LA, Olsen A, et al. The DNA sequence and biology of human chromosome 19. Nature 2004;428:529-535.

29 Shaw CJ, Lupski JR. Implications of human genome architecture for rearrangement-based disorders: the genomic basis of disease. Hum Mol Genet 2004;13 (Spec No 1):R57-R64.

30 Klein WM, Molmenti EP, Colombani PM, et al. Primary liver carcinoma arising in people younger than 30 years. Am J Clin Pathol 2005;124:512-518.

31 Morise Z, Sugioka A, Mizoguchi Y, et al. Fibrolamellar carcinoma of the liver in a Japanese hepatitis B virus carrier. J Gastroenterol Hepatol 2005;20:1136-1138.

32 Dadke D, Jaganath $\mathrm{P}$, Krishnamurthy $\mathrm{S}$, et al. The detection of HBV antigens and HBx-transcripts in an Indian fibrolamellar carcinoma patient: a case study. Liver 2002;22:87-91.

33 Davison FD, Fagan EA, Portmann B, et al. HBV-DNA sequences in tumor and nontumor tissue in a patient with the fibrolamellar variant of hepatocellular carcinoma. Hepatology 1990;12:676-679.

34 Vecchio FM, Fabiano A, Ghirlanda G, et al. Fibrolamellar carcinoma of the liver: the malignant counterpart of focal nodular hyperplasia with oncocytic change. Am J Clin Pathol 1984;81:521-526.

35 He L, Tian DA, Li PY, et al. Mouse models of liver cancer: progress and recommendations. Oncotarget 2015;6:23306-23322.

36 De Minicis S, Kisseleva T, Francis $\mathrm{H}$, et al. Liver carcinogenesis: rodent models of hepatocarcinoma and cholangiocarcinoma. Dig Liver Dis 2013;45: 450-459.

37 Fausto N, Campbell JS. Mouse models of hepatocellular carcinoma. Semin Liver Dis 2010;30:87-98.

38 Bettini G, Marcato PS. Primary hepatic tumours in cattle. A classification of 66 cases. J Comp Pathol 1992;107:19-34. 